\title{
A Predictive Controller for a Heaving Buoy Producing Potable Water
}

\author{
Giorgio Bacelli, Jean-Christophe Gilloteaux and John Ringwood \\ Dept. of Electronic Engineering, \\ National University of Ireland Maynooth (NUIM) \\ gbacelli@eeng.nuim.ie
}

\begin{abstract}
A model predictive controller for a wave-powered desalination unit is presented. The desalination unit is composed of a reverse osmosis membrane, and the water is pumped at high pressure through the membrane by a wave energy converter. The wave energy converter is a heaving buoy type connected to an hydraulic circuit that converts the oscillating motion of the buoy into a rectified sea-water flow at high pressure. The controller acts on a throttle valve to regulate the pressure in the reverse osmosis unit in order to avoid damage to the membrane caused by excessive pressure and to improve both its longevity and the overall efficiency of the device. The control is constrained due to the rate and position limits of the throttle valve and the recovery ratio, which is the ratio between the total amount of pumped sea-water and the potable water. The system is also switched and non-stationary because of the check valves used for the rectification of the sea-water flow and the variation of the excitation force dynamic over time, respectively. Results are presented using a nonlinear simulation model for the plant, using both monochromatic and real sea states.
\end{abstract}

\section{INTRODUCTION}

Desalination is an essential step in potable water production from seawater; it is currently achieved by means of several techniques, among which the most common are distillation, ion exchange and membrane processes. Reverse Osmosis (RO) is a membrane-type process in which water with high salt concentration is forced through a semipermeable membrane that restrains the passage of salt.

Since the first experiments in the late 1950s by Reid and Breton [1] and the first practical application in early 1960s by Loeb and Sourirajan [2], RO has achieved the largest installed desalting capacity throughout the world [3], mainly because of the inherent simplicity of the process and the relatively high energy efficiency.

Water is forced through the membrane by the application of pressure. The pressure drop across the membrane is proportional to the salinity of the water and is called osmotic pressure. Part of the pressurized sea water has to be discarded in order to avoid extremely high salt concentration that can result in excessive increase of the osmosis pressure and malfunctioning of the membrane. The ratio between the desalinated water volume and the total sea water volume pumped into the circuit is called the recovery ratio. Energy is lost with discarded pressurized sea water and, generally, Energy Recovery Devices (ERD) are used to increase the efficiency. A review of desalination processes powered by Wave Energy Converters (WEC) is provided by Davies in [4], where a number of applications are described, some of which are already operational. WECs provide a variable source of energy; therefore, it is necessary to stabilize the pressure. The motivation for the design of a constant pressure controller is to minimize the specific energy consumption of the device, especially if ERDs are used as described in [5] and maximize the longevity of the membrane [6]. Besides, this type of control prevents damage to the unit caused by excessive pressure.

Fig. 1 depicts the WEC considered; it is a heaving buoy type or point absorber. A comprehensive description of this device is provided in [7], while a different approach for pressure regulation is described in [8].

The alternating vertical motion of the device pumps water into the hydraulic circuit. The water flow is then rectified by four check valves, smoothed by a gas accumulator and filtered for particles. As shown in Fig. 2, a fraction $\left(Q_{w}\right)$ of the pressurized sea water $\left(Q_{f}\right)$ flows through the membrane, and the rest flows through a throttle valve $\left(Q_{t v}\right)$ used for RO pressure regulation $\left(P_{r_{o}}\right)$. The throttle valve is subject to a rate limit (max opening or closing speed) and to position limits (fully open and fully closed). There is also a limit to the maximum value of the recovery ratio $Q_{w} / Q_{f}$.

The objective is to regulate the pressure in the reverse osmosis unit to a value of 60 bar; thus the control problem is a constrained regulation problem. Model predictive control is chosen because of its ability to deal with constrained control problems. For control purposes, both the hydrodynamic model and the hydraulic circuit model are linearized, but because of the check valves in the hydraulic circuit, the resulting model is a hybrid system in which the state space is divided in two regions, each one characterized by a linear model. The sea water flow $\left(Q_{f}\right)$ through the hydraulic circuit depends on the motion of the device that, in turn, depends on its interaction with the sea; therefore the flow $Q_{f}$ dynamically changes depending on the sea state, making the system non-stationary.

In summary, the problem is pressure regulation inside the $\mathrm{RO}$ unit $\left(P_{r_{o}}\right)$ of a system which is:

- PieceWise Linear (PWL): due the check valves in the hydraulic circuit;

- constrained: due to the recovery ratio, rate and position limit of the throttle valve;

- non-stationary: due to the variability of the sea state.

PWL systems are a subset of PieceWise Affine (PWA) systems; description and solutions for control problems of PWA and constrained PWA systems have been addressed 


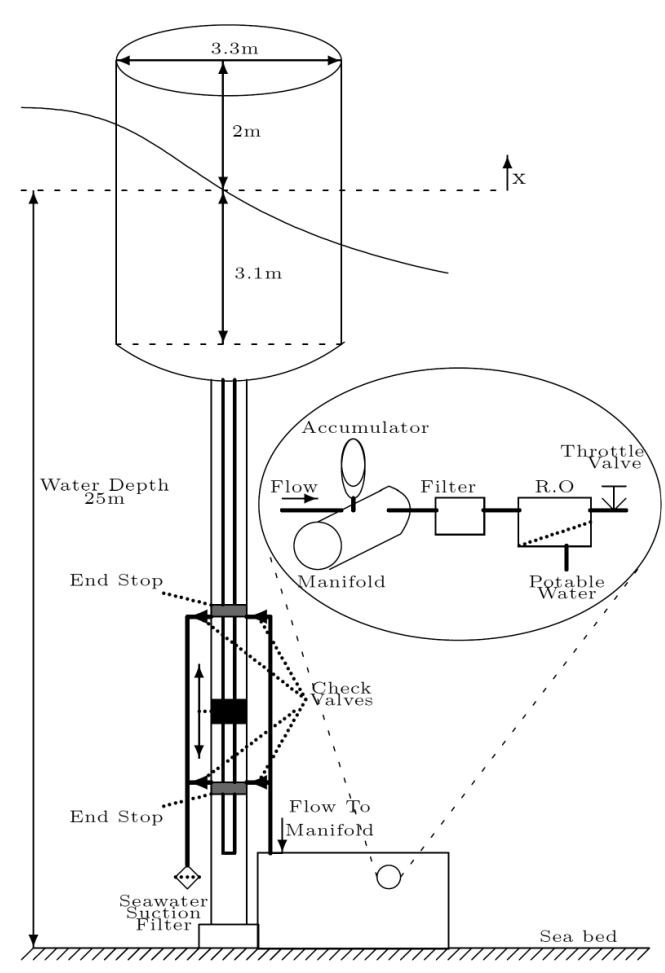

Fig. 1: WEC Diagram [8]

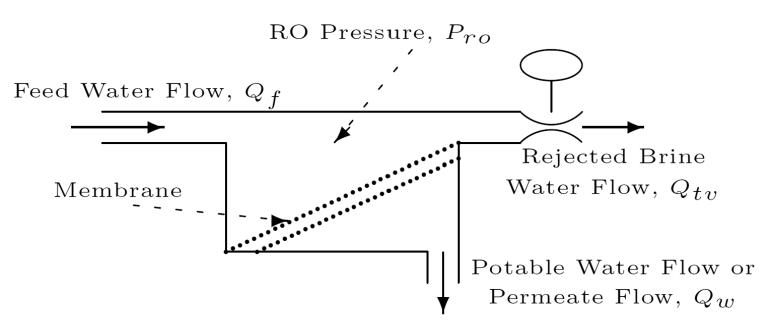

Fig. 2: RO unit diagram [8]

in the literature, for example in [9], [10] and [11], while the control of a non-stationary and constrained PWL system still constitutes a significant challenge.

The block diagram of the system is depicted in Fig. 3; the control strategy presented is based on model predictive control. A nonlinear model is used for the simulation of the hydraulic circuit, while the hydrodynamic model is based on linear wave theory. The excitation force is recursively estimated and predicted using an Auto Regressive (AR) model; it is then merged with the plant linearized model to form an augmented PWL model. A sequence of trajectories for the control input (throttle valve) is generated and then computed with the PWL model. The trajectory that satisfies the constraints and minimizes the mean square error of the pressure from the set point over the control horizon is chosen.

Section II provides the description of the nonlinear model used for the plant simulation. The control algorithm and the linearized model used for its computation are described re-

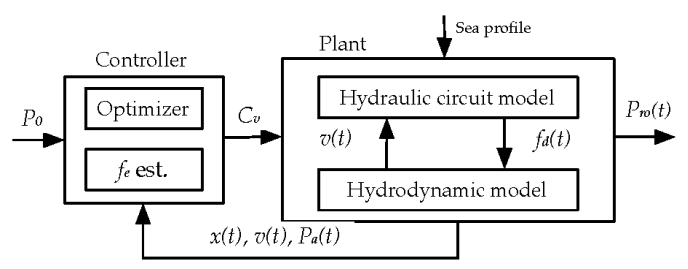

Fig. 3: System block diagram

spectively in sections IV and III, while the simulation results are provided in section $\mathrm{V}$ for both a monochromatic sea profile and real sea elevation data recorded by a wave raider buoy. Section VI contains a discussion about the controller implementation, the results, and possible improvements.

\section{NONLINEAR PLANT SIMULATION MODEL}

As depicted in Fig. 3, the plant simulation model computes the motion of the buoy and the hydraulic circuit as a function of the sea profile and the control signal. In particular, it provides the vertical velocity and position of the buoy and the water pressure inside the hydraulic circuit (respectively $\left.v(t), x(t), P_{a}(t)\right)$ as functions of the throttle valve position $\left(C_{v}\right.$, set by the controller) and of the sea profile. The choice of variables provided by the model is motivated by the fact that they can easily be accessed in a real system by means of commonly available sensors. The model is divided in two block, one describing the buoy motion (sec. II-A) and the other describing the water pressure inside the gas accumulator and in the RO unit (sec. II-B).

\section{A. Hydrodynamic model}

The oscillating motion of a floating body is generally described by means of Newton's second law $\mathbf{F}_{t o t}=m \mathbf{a}$, where $m$ is the buoy mass and $\mathbf{a}$ is the acceleration vector caused by the application of the force $\mathbf{F}_{t o t}$. The total applied force $\left(\mathbf{F}_{t o t}\right)$ is the combination of the forces caused by the interaction of the body with the sea and the hydraulic circuit. It is considered the simple case of a body with a single degree of freedom oscillating in heave. The frequency domain description of the interaction between body and fluid is described by eq. (1). The total force acting on the body $\left(F_{t}(\omega)\right)$ is a function of the body velocity fourier transform $(H(\omega))$ and the sea profile fourier transform $(U(\omega))$ [12].

$$
F_{t}(\omega)=F_{e}(\omega) H(\omega)-Z_{r}(\omega) U(\omega)
$$

The term $F_{e}(\omega) H(\omega)$ on the right hand side of (1) is the excitation force where $F_{e}(\omega)$ is the excitation force coefficient matrix, while $Z_{r}(\omega) U(\omega)$ is the radiation force with $Z_{r}(\omega)$ the radiation impedance matrix. Since the system considered is characterized by only one degree of freedom (heave), all the terms in (1) are scalar functions of the angular frequency $(\omega)$. The excitation force coefficient and the radiation impedance are functions of the body geometry; their values are generally obtained by wave tank experiments or by numerical solution of the radiation and excitation boundary-value problems on the mean wet body surface. The 
hydrodynamic coefficients used here have been calculated using the WAMIT commercial software package.

The time domain formulation of (1), required for the numerical integration of Newton's second law, is given by:

$$
\begin{aligned}
f_{t}(t)= & \int_{-\infty}^{\infty} \eta(\tau) f(t-\tau) d \tau+ \\
& -m_{r}(\infty) \ddot{x}(t)-\int_{-\infty}^{t} k(t-\tau) \dot{x}(\tau) d \tau
\end{aligned}
$$

where $\eta(t)$ is the sea profile, $\dot{x}(t)$ and $\ddot{x}(t)$ are the vertical velocity and acceleration of the buoy respectively; $f(t)$ is the inverse fourier transform of $F_{e}(\omega)$, that is, the impulse response for the excitation force. The time domain description of the radiation force is given by the last two terms of eq.(2) [13] where:

$$
\begin{aligned}
k(t) & =\frac{2}{\pi} \int_{0}^{\infty} \operatorname{Re}\left[Z_{r}(\omega)\right] \cos (\omega t) d \omega, \\
m_{r}(\infty) & =\lim _{\omega \rightarrow \infty} \operatorname{Im}\left[Z_{r}(\omega)\right] .
\end{aligned}
$$

The efficient computation of the convolution product can be improved by considering it as the response of a linear system described by the matrices $\left(\mathbf{A}_{\mathbf{r}}, \mathbf{B}_{\mathbf{r}}, \mathbf{C}_{\mathbf{r}}, \mathbf{D}_{\mathbf{r}}\right)$ [14]. This is performed by system identification via a Hankel singular value decomposition [15], as in [16]. The radiation force $\left(f_{r}(t)\right)$ is then expressed as:

$$
\begin{aligned}
\dot{\mathbf{x}}_{\mathbf{r}}(t) & =\mathbf{A}_{\mathbf{r}} \mathbf{x}_{\mathbf{r}}(t)+\mathbf{B}_{\mathbf{r}} \dot{x}(t) \\
f_{r}(t) & =\mathbf{C}_{\mathbf{r}} \mathbf{x}_{\mathbf{r}}(t)+D_{r} \dot{x}(t)
\end{aligned}
$$

Additional terms for the description of the hydrostatic stiffness $\left(f_{s}(t)\right)$ and friction $\left(f_{f}(t)\right)$ are also included :

$$
f_{s}(t)=-S x(t), \quad f_{f}(t)=R_{f} \dot{x}(t) .
$$

\section{B. Hydraulic circuit}

As depicted in the hydraulic circuit diagram of Fig. 1, the alternating motion of the buoy pressurizes water, which flows into the hydraulic circuit. The alternating flow is rectified by means of four check valves that cause a pressure drop $(\Delta P)$ dependent on the flow $Q$ as in (5), which is the relation between pressure and laminar flow through an orifice:

$$
Q=C_{v_{1}} \sqrt{\Delta P}
$$

where $C_{v_{1}}$ is the check valve flow coefficient. The same relation between flow and pressure drop is used for the modelling of the throttle valve and the particle filter.

An isentropic transformation of a perfect gas is considered for the charging and discharging of the gas accumulator; the water volume stored $\left(V_{a}\right)$ and its pressure $\left(P_{a}\right)$ are related as:

$$
P_{a}\left(V_{a}\right)=\frac{P_{0}}{\left(1-\frac{V_{a}}{V_{0}}\right)^{\gamma}}
$$

where $\gamma$ is the heat capacity ratio, $V_{0}$ the accumulator volume and $P_{0}$ the pre-charge pressure.
The volume of the water stored in the accumulator is modelled by (7), which is obtained writing the flow balance at the manifold (Fig.1):

$$
\dot{V}_{a}(t)=|\dot{x}(t)| A-C_{v_{5}} \sqrt{P_{a}\left(V_{a}\right)-P_{r o}\left(V_{a}, C_{v}\right)}
$$

The first term on the right hand side of (7) is the sea water flow pumped into the hydraulic circuit by the motion of the buoy, which is the absolute value of the velocity $(\dot{x}(t))$ multiplied by the area of the pump $(A)$. The last term on the right hand side is the flow through the particle filter characterized by the flow coefficient $C_{v_{5}}$, which is also the flow entering into the $\mathrm{RO}$ unit. This flow depends on the accumulator pressure $\left(P_{a}\right)$ and on the throttle valve flow coefficient $\left(C_{v}\right)$, which affects the RO pressure $\left(P_{r o}\left(V_{a}, C_{v}\right)\right)$. The flow balance equation for the RO unit depicted in Fig. 2 is:

$$
\begin{aligned}
C_{v_{5}} & \sqrt{P_{a}\left(V_{a}\right)-P_{r o}\left(V_{a}, C_{v}\right)}= \\
& =\left(P_{r_{o}}\left(V_{a}, C_{v}\right)-P_{o s m}\right) \rho_{r o}+C_{v_{5}} \sqrt{P_{r o}\left(V_{a}, C_{v}\right)}
\end{aligned}
$$

where $P_{o s m}$ and $\rho_{\text {ro }}$ are the parameters characterizing the RO membrane; they are, respectively, the osmosis pressure and the permeability coefficient.

The influence of the hydraulic circuit on the motion of the device is described by eq. (9), which is the damping force exerted by the hydraulic pump on the buoy.

$$
f_{d}(t)=\operatorname{sign}(v(t)) A\left(P_{a}(t)+\left(\frac{|\dot{x}(t)| A}{C_{v_{1}}}\right)^{2}\right)
$$

The first term inside the parenthesis is due to pressure in the accumulator, while the second is due to the pressure drop across the check valves. The term $\operatorname{sign}(v(t))$ is introduced by the check valves and indicates that the direction of this force is always opposite to the velocity (it opposes the motion of the buoy).

\section{Complete model}

The complete nonlinear model for the WEC is described by (10)-(14). It is composed of three first-order differential equations plus an additional first-order differential equation (13) coupled with an algebraic equation (14).

$$
\begin{aligned}
\dot{x}(t)= & v(t) \\
\dot{v}(t)= & \frac{1}{m+m_{r}(\infty)}\left[f_{e}(t)-\mathbf{C}_{\mathbf{r}} \mathbf{x}_{\mathbf{r}}(t)-D_{r} v(t)+\right. \\
& -\operatorname{sign}(v(t)) A\left(P_{a}(t)+\left(\frac{|v(t)| A}{C_{v_{1}}}\right)^{2}\right)+ \\
& \left.-S x(t)-R_{f} v(t)\right] \\
\dot{\mathbf{x}}_{\mathbf{r}}(t)= & \mathbf{A}_{\mathbf{r}} \mathbf{x}_{\mathbf{r}}(t)+\mathbf{B}_{\mathbf{r}} v(t) \\
\dot{V}_{a}(t)= & |v(t)| A-C_{v_{5}} \sqrt{P_{a}-P_{r o}} \\
& C_{v_{5}} \sqrt{P_{a}-P_{r o}}= \\
= & \left(P_{r o}-P_{o s m}\right) \rho_{r o}+C_{v} \sqrt{P_{r o}}
\end{aligned}
$$




\section{MODEL LINEARIZATION}

The model in (10)-(14) is linearized [7] in order to reduce the complexity of the controller model, therefore reducing the computation time. The PWL system resulting from the linearization is:

$$
\begin{aligned}
\dot{\mathbf{x}}_{\mathbf{b}}(t) & =\mathbf{A}_{\mathbf{b}} \mathbf{x}_{\mathbf{b}}(t)+\mathbf{B}_{\mathbf{b}} C_{v}(t)+\mathbf{B}_{\mathbf{b}_{\mathbf{e}}} f_{e}(t) \\
\mathbf{x}_{\mathbf{b}}(t) & =[x(t) v(t) P(t)]^{T} \\
\mathbf{A}_{\mathbf{b}} & =\left[\begin{array}{ccc}
0 & 1 & 0 \\
-\frac{S}{M} & \frac{R_{f}+K_{s t}}{M} & -\alpha \frac{A}{M} \\
0 & \alpha \frac{A}{C_{A c c}} & -\rho_{r o} \frac{\overline{P_{r o}}-P_{o s m}}{C_{A c c} \overline{P_{r o}}}
\end{array}\right]
\end{aligned}
$$

with:

$$
\mathbf{B}_{\mathbf{b}}=\left[\begin{array}{c}
0 \\
0 \\
\frac{-C_{v_{\max }} \sqrt{\overline{P_{r o}}}}{200 C_{A c c}}
\end{array}\right], \quad \mathbf{B}_{\mathbf{b}_{\mathbf{e}}}=\left[\begin{array}{c}
0 \\
1 / M \\
0
\end{array}\right]
$$

The state variables that constitute the state vector $\left(\mathbf{x}_{\mathbf{b}}(t)\right)$ of the linearized system are the vertical position $(x(t))$, the velocity $(v(t))$ and the accumulator pressure $(P(t)) ; M=$ $m+m_{r}(\infty), K_{s t}$ is the static approximation of the radiation kernel $k(t), C_{a c c}$ is the accumulator capacitance, $C_{v_{\max }}$ is the flow coefficient of the throttle valve in its fully open position, and $\overline{P_{\text {ro }}}$ is the nominal RO pressure $(60$ bar $)$. The parameter $\alpha=\operatorname{sign}(v(t))$ describes the switching of the system.

\section{A. Excitation force model estimation}

With the reasonable assumption of measurable state variables $(x(t), v(t), P(t))$, the excitation force $f_{e}(t)$ can be calculated directly from (11) and modeled by means of an Auto Regressive model of the third order (AR(3)), computed with a recursive least square algorithm using a forgetting factor of 0.95 ; the model is then converted into its controller canonical form:

$$
\begin{aligned}
\mathbf{x}_{\mathbf{e}}(t+1) & =\mathbf{A}_{\mathbf{e}} \mathbf{x}_{\mathbf{e}}(t) \\
\hat{f}_{e}(t) & =\mathbf{C}_{\mathbf{e}} \mathbf{x}_{\mathbf{e}}(t)
\end{aligned}
$$

\section{B. Augmented model}

The continuous time system $\left(\mathbf{A}_{\mathbf{b}}, \mathbf{B}_{\mathbf{b}_{e}}, \mathbf{B}_{\mathbf{b}}\right)$ is discretized into $\left(\mathbf{A}_{\mathbf{b}}^{\mathbf{d}}, \mathbf{B}_{\mathbf{b}_{\mathbf{e}}}^{\mathbf{d}}, \mathbf{B}_{\mathbf{b}}^{\mathbf{d}}\right)$, then merged with (17), giving the augmented PWL model:

$$
\left[\begin{array}{c}
\mathbf{x}_{\mathbf{e}}(t+1) \\
\hdashline \mathbf{x}_{\mathbf{b}}(t+1)
\end{array}\right]=\left[\begin{array}{c:c}
\mathbf{A}_{\mathbf{e}} & \mathbf{0} \\
\hdashline \mathbf{B}_{\mathbf{b}_{\mathbf{e}}}^{\mathbf{d}} \mathbf{C}_{\mathbf{e}} & \mathbf{A}_{\mathbf{b}}^{\mathbf{d}}
\end{array}\right]\left[\begin{array}{c}
\mathbf{x}_{\mathbf{e}}(t) \\
\hdashline \mathbf{x}_{\mathbf{b}}(t)
\end{array}\right]+\left[\begin{array}{c}
\mathbf{0} \\
\hdashline \mathbf{B}_{\mathbf{b}}^{\mathbf{d}}
\end{array}\right] C_{v}(t)
$$
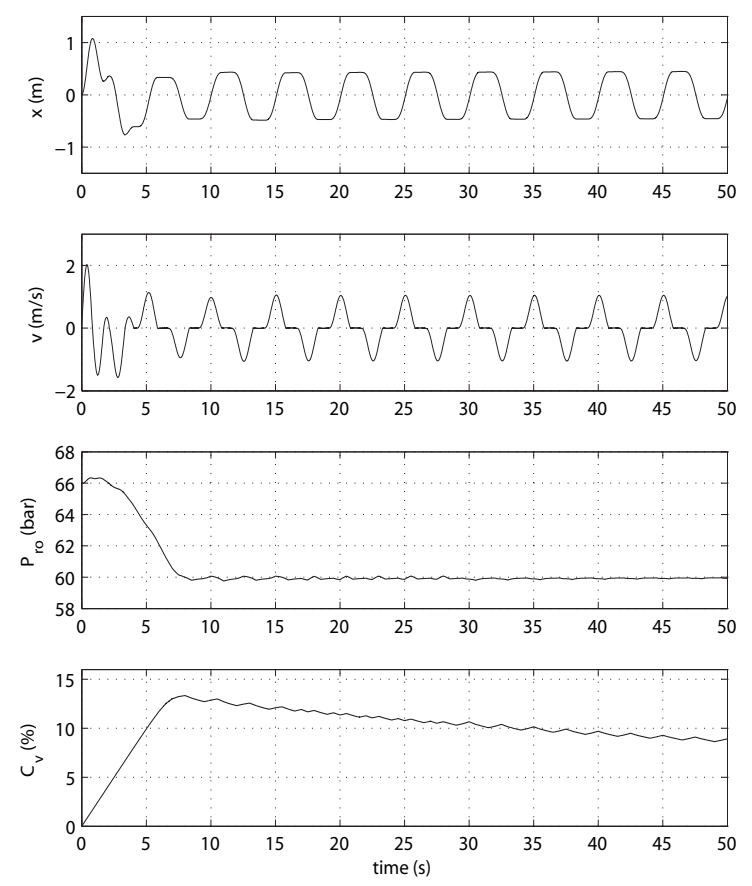

Fig. 4: Monochromatic sea profile simulation

\section{CONTROL ALGORITHM}

In order to satisfy rate and position constraints, a control trajectory of the form

$$
u(t+1)=u(t)+\delta \cdot g
$$

is generated, where

$$
\delta \in \Delta=\{-1,-0.75,-0.5,-0.25,0,0.25,0.5,0.75,1\}
$$

and $g$ is a gain term, such as:

$$
\beta \cdot \Delta u_{\max }<g<\Delta u_{\max }, \quad 0<\beta<1
$$

that depends on the output error $P_{r o}-P_{0}$ as:

$$
g=\left(\beta+(1-\beta)\left(1-e^{-\left|P_{r o}-P_{0}\right|}\right)\right) \cdot \Delta u_{\max }
$$

A generic trajectory $\left[u(t+1), u(t+2), \ldots, u\left(t+n_{s}\right)\right]$ where $n_{s}$ is the prediction horizon, is calculated as:

$$
\begin{aligned}
u(t+1)= & u(t)+\delta_{1} \cdot g \\
u(t+2)= & u(t+1)+\delta_{2} \cdot g \\
\vdots & \vdots \\
u\left(t+n_{s}\right)= & u\left(t+n_{s}-1\right)+\delta_{n s} \cdot g
\end{aligned}
$$

with: $\quad \delta_{1}, \delta_{2}, \ldots, \delta_{n s} \in \Delta$

A set of trajectories $\left(U_{T}\right)$ is then generated considering all the $N$ possible combinations of $\delta_{1}, \delta_{2}, \ldots, \delta_{n s}$, and it is stored in the matrix $\mathbf{U}$ : 

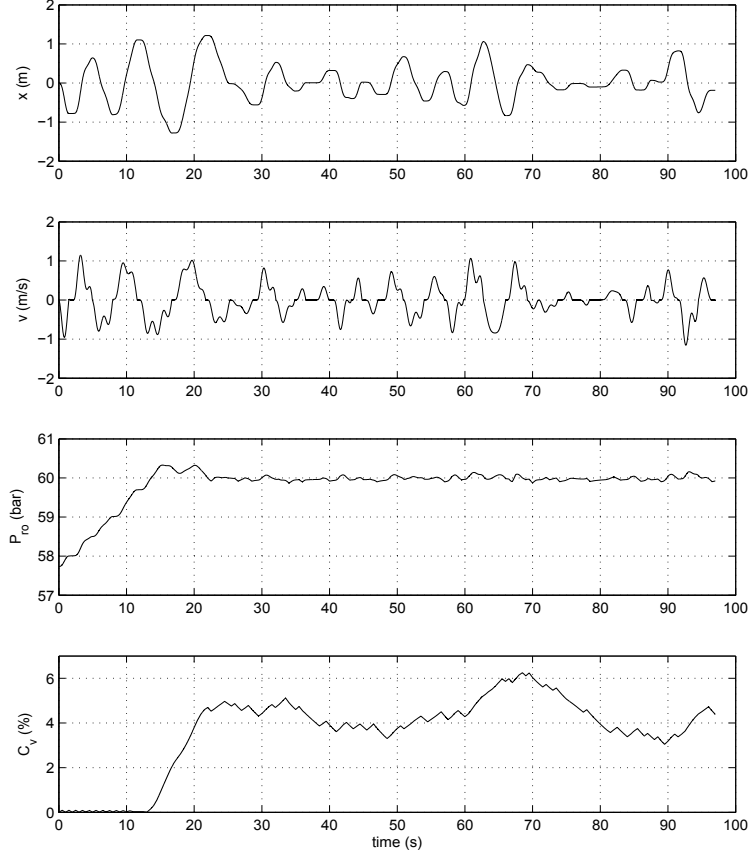

Fig. 5: Measured sea profile simulation with initial pressure lower than the set point

$$
\mathbf{U}=\left[\begin{array}{ccc}
u_{1}(t+1) & \ldots & u_{1}\left(t+n_{s}\right) \\
\vdots & \ddots & \vdots \\
u_{N}(t+1) & \ldots & u_{N}\left(t+n_{s}\right)
\end{array}\right]
$$

where $N=|\Delta|^{n_{s}}$.

The augmented PWL system in (18) is simulated $N$ times, from $t+1$ to $t+n_{s}$, each time assigning a row of $U$ as a control sequence for the input $C_{v}($.$) . At the same time,$ the position and the recovery ratio constraints are verified, providing a set $U_{A} \subseteq U_{T}$ of allowed trajectories. From all the allowed trajectories $\left(U_{A}\right)$, the controller selects the one that minimizes:

$$
\min _{i \in N}\left\|\left[P_{i}(t+1) P_{i}(t+2) \ldots P_{i}\left(t+n_{s}\right)\right]-P_{0}\right\|_{2}
$$

which is the mean square error from the set point $P_{0}$ of the pressure $P_{i}($.$) , where P_{i}($.$) is the pressure profile from t+1$ to $t+n_{s}$ corresponding to the control sequence $u_{i}(.) \in U_{A}$. If no allowed trajectories are found $\left(U_{A}=\emptyset\right)$, the controller stops the device because it is likely that either the sea is too calm and not enough power is available, or the sea is too strong for the the device to be operational. The control input provided to the plant at the time $t$ is then the first element of the control sequence $u_{i}($.$) .$

\section{RESULTS}

The plant simulation model is defined in continuous time, and the differential equations (10)-(13) are solved numerically using the fourth-order Runge-Kutta method with a time step of $0.01 s$. The controller is computed with a time step of $0.5 \mathrm{~s}$ and a control horizon of $n_{s}=2$. The control input is provided as a percentage (\%) of the fully open position. Therefore, the position and rate limit of the throttle valve are, respectively, $0<u<100 \%$ and $\Delta u_{\max }=10 \% / \mathrm{s}$. In eq. (21), the parameter $\beta=0.1$ and the maximum recovery ratio $Q_{w} / Q_{f}$ is 0.35 . The system has been tested for both a monochromatic sea state and using sea profile data measured by a wave rider buoy. A monochromatic sea profile with a period of $T=5 \mathrm{~s}$ has been used for the first simulation, and the results for the motion of the device $(x(t), v(t))$, for the controlled variable $P_{r_{o}}$ and for the control input $C_{v}$ are plotted on Fig.4. Real sea elevation data have been used to simulate the response of the system, with the control action based on the predicted excitation force $\left(\hat{f}_{e}(t)\right)$. For comparison, the response of the system when the controller uses the true excitation force $\left(f_{e}(t)\right)$ has been simulated. The results of these two simulations are plotted in Fig. 6 , where $P_{r o}^{e}$ and $C_{v}^{e}$ are, respectively, the system output and the control signal when the controller uses $\hat{f}_{e}(t) ; P_{\text {ro }}$ and $C_{v}$ are the system output and the control signal when the controller uses $f_{e}(t)$ respectively. Fig.6 also shows the excitation force $\left(f_{e}(t)\right)$ vs its two-step ahead $(1 s)$ prediction $\left(\hat{f}_{e}(t)\right)$. A simulation with a different initial condition (initial pressure lower than the pressure set point), using the same set of sea elevation data, is depicted in Fig. 5.

\section{CONCLUSIONS}

A predictive control strategy for a switching, constrained and non-stationary control problem, using a nonlinear plant simulation model have been implemented. The control algorithm computes the response of the PWL system for a set of control signal trajectories, selecting the one that satisfies the constraints and minimizes the mean square error of the pressure from the set point. The control input increment, $\Delta u$, is restricted to take values from the finite set $\Delta$, and this can result in a coarse regulation of the output; this effect can be reduced increasing the number of the elements of $\Delta$, causing an increase in the computation time. The gain $g$ has been introduced to improve the control performance without affecting the computational effort; it refines the control action when the output error is small and viceversa. Restricting $\Delta u$ to take a value from a finite set is not a strong limitation because this happens every time a digital control is interfaced with a real system (A/D and D/A conversion). The set $\Delta$ has been built with uniformly distributed values between -1 and 1 (20). Future work will involve the distribution optimization for these elements, the study of a better choice for the gain function (21) and the study of stability and robustness properties.

\section{ACKNOWLEDGMENTS}

The authors gratefully acknowledge the financial support of Enterprise Ireland under contract EI/CTFD/2006/IT/325.

\section{REFERENCES}

[1] C. E. Reid and E. J. Breton, "Water and ion flow across cellulosic membranes," Journal of Applied Polymer Science, vol. 1, no. 2, pp. 133-143, 1959. 

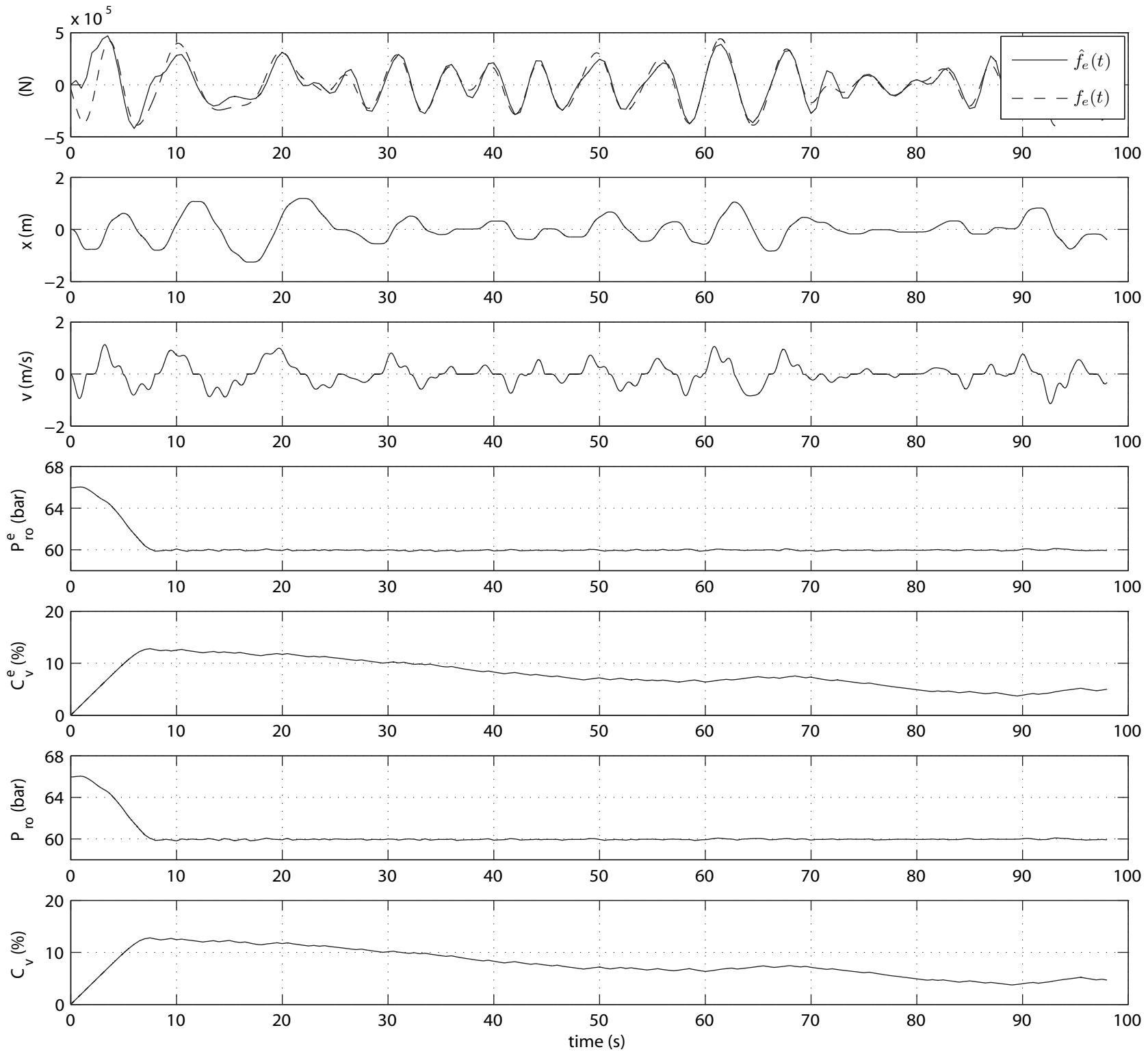

Fig. 6: Measured sea profile simulation with initial pressure higher than the set point

[2] S. Loeb and S. Sourirajan, "Sea water demineralization by means of an osmotic membrane,," Advances in Chemistry Series, vol. 38, pp. 117132, 1963.

[3] Z. Yuan and R. S. Tol, "Evaluating the costs of desalination and water transport," Working Paper FNU-41, Research Unit Sustainability and Global Change, Hamburg University, June 2004.

[4] P. Davies, "Wave-powered desalination: resource assessment and review of technology," Desalination, vol. 186, pp. 97-109, Dec. 2005.

[5] M. Folley, B. Peate Suarez, and T. Whittaker, "An autonomous wavepowered desalination system," Desalination, vol. 220, pp. 412-421, Mar. 2008.

[6] J. Cruz, ed., Ocean Wave Energy: Current Status and Future Perspectives. Springer-Verlag, 2008.

[7] G. Nolan and J. Ringwood, "Control of a heaving buoy wave energy converter for potable water production," in Irish Signals and Systems Conference ISSC 2006, June 2006.

[8] G. Nolan, Modelling and Optimisation of a Heaving Buoy Wave Energy Converter for Potable Water Production. PhD thesis, National University of Ireland, Maynooth, 2006.

[9] E. Sontag, "Nonlinear regulation: The piecewise linear approach,"
IEEE Transactions on Automatic Control, vol. 26, no. 2, pp. 346-358, 1981.

[10] D. Q. Mayne and S. Rakovic, "Model predictive control of constrained piecewise affine discrete-time systems," International Journal of Robust and Nonlinear Control, vol. 13, no. 3-4, pp. 261-279, 2003.

[11] F. Borrelli, M. Baotic, A. Bemporad, and M. Morari, "Dynamic programming for constrained optimal control of discrete-time linear hybrid systems," Automatica, vol. 41, pp. 1709-1721, Oct. 2005.

[12] J. Falnes, Ocean waves and oscillating systems: linear interactions including wave-energy extraction. Cambridge University Press, 2002.

[13] W. Cummins, "The impulse response function and ship motions," Schiffstechnik, vol. 9, pp. 101-109, 1962.

[14] Z. Yu and J. Falnes, "State-space modelling of a vertical cylinder in heave," Applied Ocean Research, vol. 17, pp. 265-275, Oct. 1995.

[15] S. Kung, "A new identification and model reduction algorithm via singular value decompositions," in Proc.Twelth Asilomar Conf. on Circuits, Systems and Computers, 1978.

[16] E. Kristiansen, A. Hjulstad, and O. Egeland, "State-space representation of radiation forces in time-domain vessel models," Ocean Engineering, vol. 32, pp. 2195-2216, Dec. 2005. 